\title{
Extracting data from a chemotherapy prescription platform for real-world oncology research in the UK: a pilot study
}

\author{
Laura McDonald ${ }^{1}$, Dara Stein², Robert Carroll ${ }^{1}$, Mira Soni², Anna Schultze², Sreeram \\ Ramagopalan*,1 \& John Wagstaff ${ }^{3}$ \\ ${ }^{1}$ Bristol-Myers Squibb, Uxbridge, UB8 1DH, UK \\ ${ }^{2}$ Evidera, London, W6 8BJ, UK \\ ${ }^{3}$ South West Wales Cancer Institute, Singleton Hospital, Swansea, SA2 8QA, UK \\ *Author for correspondence: Sreeram.Ramagopalan@bms.com
}

\begin{abstract}
Aim: In the UK, there are limited data sources for evaluating real-world research questions related to oncology therapy. We conducted a pilot study to investigate the feasibility of extracting data directly from a chemotherapy prescription platform (ChemoCare ${ }^{\circledR}$ ) utilized in standard care. Patients \& methods: Concordance was compared with data extracted manually for patients with advanced melanoma as part of a concurrent chart review (gold-standard) using Cohen's kappa and the intraclass correlation coefficient. Results: There was high concordance between data automatically extracted from the prescription platform and chart review data. Conclusion: This pilot can be used as a framework for future studies using direct, automated extraction from prescription platforms.
\end{abstract}

Lay abstract: It is important to study how cancer treatments are used as part of usual care. Historically, such data have been collected by manually extracting data from patient medical records. The use of prescription platforms allows the same data to be extracted automatically which reduces the risk for errors to be introduced and is time saving for clinical staff. We conducted a pilot study comparing data extracted from a prescription platform commonly used in the NHS, ChemoCare, to data extracted manually. Our findings showed that the automatically extracted data was valid for use in research.

First draft submitted: 19 March 2019; Accepted for publication: 7 May 2019; Published online:

4 June 2019

Keywords: epidemiology • immunotherapy • melanoma • skin

While the aim of clinical trials is to demonstrate the clinical efficacy and safety of a therapy within a controlled environment, studies involving real-world data (RWD) offer the opportunity to understand longer term effectiveness in broader patient populations. With the continued emergence of new therapies in oncology, RWD on current clinical practice patterns and outcomes are needed. However, in the UK, there remain limited options for evaluating oncology research questions leveraging existing data. For example, oncology drugs cannot be identified in the Clinical Practice Datalink, and the Systemic Anti-Cancer Therapy Dataset is still affected by major time lags in data availability.

In the absence of suitable existing databases in oncology, retrospective chart review studies permit the collection of RWD in a relatively short timeframe. While chart reviews are less resource-intensive compared with prospective studies, they can still be time consuming and are prone to manual abstraction errors. The burden of data collection in traditional chart review studies could be further reduced with hospital electronic medical record (EMR) systems that permit automated direct extraction of the study variables, meaning that hospital staff would no longer need to perform manual data review and entry. When assessing novel sources of RWD, careful consideration of the validity of the extracted data is needed as hospital EMR system formats vary and are not designed for research purposes.

The current pilot study extracted data on patient characteristics, treatment patterns and survival status directly from a prescription platform (ChemoCare) utilized in standard care at hospitals in the UK. The objective was to evaluate the validity of the extracted data as compared with that obtained via retrospective chart review.

Future Medicine 


\section{Patients \& methods}

\section{Study design}

A single center (Singleton Hospital, Swansea, UK) participating in a concurrent chart review study and using the prescription platform as part of usual care participated in this pilot study. The study was approved by an independent research ethics committee (REC) and the NHS Health Research Authority (NHS-HRA). Data were released from the prescription platform following Caldicott Guardian approval at Singleton Hospital, as indicated under the 'Ethical conduct of research' section.

\section{Patient inclusion \& exclusion criteria}

Adult patients ( $\geq 18$ years of age) with advanced (unresectable or metastatic) melanoma who initiated first-line therapy between 1 July 2016 and 1 July 2017 were included. Patients were excluded if they had participated in a first-line trial, previously received systemic therapy for their advanced disease, or had a concurrent active malignancy requiring treatment. We restricted the population to patients with advanced disease as we were particularly interested in capturing recently licensed gene-targeted or immune-oncology therapies, which are only indicated for this population. All eligible patients were included.

\section{Data source \& collection process}

For the chart review, local site staff identified patients, abstracted data from their hospital medical charts (EMR/paper records), and manually entered the data into an electronic data capture (EDC) system. This included pseudonymized (i.e., anonymous to nonsite staff) data on demographics, disease characteristics, treatment history, first-line treatment patterns, clinical outcomes, healthcare resource utilization and adverse events. No formal source data verification was carried out; however, the EDC system included built-in validation checks at the point of data entry (e.g., dates outside expected range) for quality-control purposes. A data review was carried out once data for all patients were included in the system. Where discrepancies were noted, queries were generated for resolution by site staff.

For this pilot study, the ChemoCare ${ }^{\circledR}$ platform vendor (CIS-oncology, Ltd, Coventry, UK) identified patients who met the study inclusion and exclusion criteria and performed data extraction. ChemoCare is a prescription platform used for systemic therapy prescribing and patient management in over $80 \%$ of the UK's NHS cancer centers and has been in use in the NHS for over 15 years [1]. CIS-oncology assigned the same unique identifiers utilized in the chart review to each patient identified in the prescription platform and transferred the pseudonymized data from the prescription platform for analysis using secure file transfer software (File Transfer Protocol [FTP]). The de-identified data provided by CIS-oncology included demographic variables (e.g., age, sex and body mass index $[\mathrm{BMI}]$ ), disease characteristics (e.g., cancer type and stage at therapy initiation), systemic treatment patterns (e.g., treatment type, cycle dates) and survival status (e.g., date of death). The data-collection end date from the chart review study was applied to the prescription platform data to ensure consistent follow-up periods in both datasets and facilitate comparison of results.

\section{Variable definitions}

Age and gender were extracted directly from the platform. BMI was estimated based on weight and height records recorded at the start of each drug cycle. The values used in our analyses were taken at the start of first-line therapy initiation; if these were missing, records from the initiation of adjuvant therapy were used (in patients with prior therapy). Time from diagnosis to first-line therapy initiation was estimated based on the diagnosis date and date of therapy initiation, both of which were automatically extracted.

Lines of therapy were defined by the presence of a treatment switch, where each switch was taken to indicate the start of a different line. The date of discontinuation was inferred through the cycle orders; discontinuation was defined as having occurred either if there were no future cycle orders after the study end-date, if the patient switched treatment, or died. This definition was implemented to align with the definition used in the concurrent chart review. Date of death was extracted from the platform in order to estimate overall survival.

\section{Data analysis}

Data were first summarized descriptively as counts (n) and frequencies (\%; not reported here). As part of the analysis of the prescription platform data, data derivation rules were developed and implemented for the following 
measures: stage of disease, number of therapy lines, receipt of adjuvant therapy, number of first-line therapy cycles received, therapy line discontinuation status and time from treatment start to discontinuation.

Agreement between the data sources was compared using Cohen's kappa statistic for categorical variables and the intraclass correlation coefficient (ICC) for continuous variables using the 'irr' package in the statistical program R. The chart review data were considered the gold standard source to which the data extracted from the prescription platform were compared. In cases where data directly exported from the prescription platform and data manually extracted from the medical chart appeared to show discrepant information, the site investigator was contacted to confirm the source that was accurate and to further explore the reasons for discrepancies.

Analyses were performed using SAS software, Version 9.4 (SAS Institute Inc., NC, USA) and RStudio Version 1.0.153 (RStudio, Inc., MA, USA).

\section{Results}

\section{Patient identification}

Data were extracted from 37 patients in total. All patients included in the chart review were identified in the prescription platform $(n=26)$. Further, 11 additional patients in the prescription platform were found to fulfill the inclusion and exclusion criteria of the study (11/37; 29.7\%); these patients had not been identified during manual review of charts at the site, and were therefore not included in the original chart review. One patient in the prescription platform was noted as having sarcoma rather than melanoma; upon discussion with the site, it was confirmed that the most up-to-date diagnosis was melanoma.

\section{Concordance between data sources}

There was generally a high concordance between the prescription platform and information taken directly from medical notes, including demographic variables, disease characteristics, systemic treatment patterns, treatment discontinuation and survival status (Table 1).

Age, BMI, time from treatment start to discontinuation, time from treatment start to death and the number of first-line cycles all showed very good concordance (ICC $=1.00,0.99,0.97,1.00$ and 1.00 respectively); however, agreement in terms of time since diagnosis at first-line initiation was poor; with the ICC statistic not possible to reliably estimate. Most categorical variables considered showed perfect concordance (Cohen's kappa $=1$ ); however, it was not possible to calculate the concordance for disease stage due to a lack of patients considered as stage IIIC in ChemoCare resulting in a lack of category overlap between the data sources.

\section{Discussion}

Direct extraction of data from electronic platforms is an efficient alternative to traditional chart review studies, as it bypasses the need for manual abstraction [2]. As a result, the data may be more accurate, that is, less prone to abstraction errors compared with manual chart review which can lead to more accurate identification of eligible patients. This was confirmed in our study, where the sample size of patients from the participating site was almost a third larger when using the automated prescription platform extraction to identify participants, compared with manual screening of patients notes.

When formally assessing the concordance between variables recorded on the platform and the manually extracted chart review data, we found high concordance for most variables. One noted discrepancy between data held in the prescription platform and the gold-standard chart review data was that the duration between advanced diagnosis and first-line therapy initiation extracted from the prescription platform was generally shorter than that reported in the chart review. This was investigated and confirmed to be because the site staff only entered into the prescription platform the most recent (advanced) diagnosis date that corresponded with decision to initiate systemic treatment rather than the initial diagnosis date of advanced disease which was captured in the chart review. This highlights the importance of understanding the structure of the data entered into hospital prescription platforms prior to analysis. For disease stage, it was not possible to formally calculate concordance statistics. This was due to a lack of overlap between categories in the databases, with three patients in the chart review being recorded as stage IIIC, whereas no patients in the prescription platform were classified as stage IIIC. However, it is important to highlight that $89 \%$ of patients were identified as being stage IV in both data sources. It should be noted that the disease stage on the prescription platform corresponds to the stage at the advanced diagnosis date which led to the initiation of treatment, and not the initial disease diagnosis date. Future studies utilizing this prescription platform for analyses 
Table 1. Concordance between data extracted from prescription platform and manual patient chart review for

demographics, clinical and disease characteristics and treatment patterns of advanced melanoma patients.

Variable

Concordance statistic

ICC $(95 \% \mathrm{CI})$

Age

$1.00(0.98-1.00)$

BMI

0.99 (0.97-0.99)

Time from diagnosis to first-line initiation

Not estimable ${ }^{\dagger}$

Time from first-line start to discontinuation

0.97 (0.95-0.99)

Time from first-line start to death

1.00 (NA)

Number of first-line cycles:

- Nivolumab + ipilimumab

1.00 (0.99-1.00)

- Pembrolizumab

Not applicable $\ddagger$

- Dabrafenib + trametinib

Not applicable $\ddagger$

Kappa

Gender

1

Stage at index:

- Stage IIIC

Not estimable ${ }^{\S}$

- Stage IV

Not estimable ${ }^{\S}$

Lines of therapy received:

- One line 1

- Two lines 1

Date of death

Prior systemic adjuvant therapy

First-line treatment:

- Nivolumab + ipilimumab 1

- Pembrolizumab 1

- Dabrafenib + trametinib 1

First-line discontinuation (yes/no):

- Overall

- Nivolumab + ipilimumab

- Pembrolizumab

- Dabrafenib + trametinib

Second-line treatment:

- Single agent trametinib 1

- Dacarbazine 1

- Nivolumab + ipilimumab 1

- Ipilimumab 1

- Pembrolizumab 1

$\dagger$ Not estimable; scale is not reliable due to low between-subject variation for this variable.

¥Number of pembrolizumab first-line cycles was not abstracted as part of the chart review and therefore concordance was not investigated. Cycle numbers are not applicable to dabrafenib

+ trametinib, which is an oral daily treatment.

${ }^{\S}$ All patients in the prescription platform dataset were stage IV and none stage IIIC, whereas three patients in the chart review dataset were stage IIIC; as a result the kappa statistic could

not be estimated.

BMI: Body mass index; ICC: Intraclass correlation coefficient.

should clearly note the time-points at which disease stage and diagnosis are captured to allow for an accurate interpretation of the data.

The use of automated hospital EMR exports in research is a relatively novel approach to data collection, and few studies have evaluated the accuracy of data in such platforms compared with manually extracted data. One recent study in Canada compared the concordance between data collected manually and automatically extracted from a system data warehouse (eCritical TRACER ${ }^{\circledR}$ ) in medical-surgical intensive care units. The researchers found high concordance between the resulting datasets [3]. Knake and colleagues also compared a large automated electronic health records database extracted from $\mathrm{EPIC}^{\circledR}$ to a manually created database at a maternity ward in Iowa. They 
reported generally high concordance rates, although notably higher error rates were observed for variables that were at least in part recorded by free text [4]. It should be noted that the accuracy of data extracted from EMRs likely varies depending on the platform used [5], as well as the specific variables that are being extracted.

There are some limitations to our pilot study that should be noted. First, we note that the data quality is dependent on the completeness and accuracy of the documentation available in the prescription platform, and that this may vary at an individual hospital level. The results of our analysis suggested robust concordance between data manually extracted from medical charts and information from the prescription platform. However, these findings represent the experience of one UK center and may not be generalizable to other hospitals in the UK who may use the software in a different manner. In addition, we cannot rule out that the congruence varies depending on the cancer type studied. For the purposes of evaluating the prescription platform extract in this study, patient charts were taken as the 'gold-standard'; however, we cannot rule out that there were also inaccuracies within this data source. It is also important to note that some clinically relevant variables are not documented in the prescription platform (e.g., date of initial/advanced diagnosis, biomarkers, treatment response and progression-free survival [PFS], surgery/radiotherapy), and this may restrict the use of this data source for some research questions. However, to overcome this limitation, a hybrid approach can be adopted whereby manual chart review is conducted in parallel with automated data extraction as a means to enrich the prescription platform export in order to fill in data gaps. There are also some variables available within the prescription platform, notably dosages, that were not evaluated in our pilot as they were not extracted as part of the concurrent chart review. Evaluating the accuracy also of these variables would be of interest for future research. One potential limitation is that the prescription platform only captures treatments administered at specific hospitals, and we therefore cannot capture treatments received at other hospitals. However, given that these are patients with advanced disease, frequent changes in the healthcare provider are unlikely. Finally, we recognize that our sample size was limited. However, this small sample size also allowed for a detailed investigation of discrepancies between the data sources through cross-checking individual records, something that would have been challenging with a larger sample size. In terms of strengths, this is one of few publications focusing explicitly on evaluating the validity of prescription platform data for use in research. We also note that as date of death is derived from a centrally available administrative field, we were able to capture date of death for all patients irrespective of where the death occurred. Although our study was set in the UK, electronic prescription and medical records are widely used in several different settings, and, with increased appreciation of the importance of generating timely real-world data on treatment use and outcomes, the use of such data in research is likely to increase. The relatively limited amount of information captured here likely means that automated extraction of data from this specific platform will not replace traditional chart reviews, however, sites participating in oncology research can save time and resources by extracting some elements automatically in a hybrid study while having confidence in the validity of the extracted data.

\section{Conclusion}

Direct extraction of data from the ChemoCare platform can produce datasets of high validity that can be used to understand questions related to the treatment and outcomes of oncology patients in the UK. Data extraction is rapid, repeatable, less error-prone and considerably less resource-intensive for clinical sites participating in realworld research compared with manual reviews of medical charts. In the future, resources saved due to the reduced abstraction time could allow for larger studies to be carried out. Furthermore, once approvals for data sharing are in place, data transfer was quick and easily repeatable, which can facilitate the evaluation of the use and effectiveness of recently approved therapies in the real-world setting. This pilot study involving direct data export from a prescription platform can be used as a framework for future RWD studies using this method.

\section{Future perspective}

The use of electronic prescriptions and electronic medical records is rapidly becoming the norm in the UK, in light of current government commitments to create a paper-free NHS by 2020. The use of such platforms provides an opportunity to conduct 'real-world' research faster, more accurately and using larger sample sizes - as data can automatically be extracted, removing the need for clinical site staff to manually extract patient data. This is a particularly exciting development in oncology, as many existing data sources do not accurately capture cancer treatments. With the increased use of technology and rapidly increasing and expanding use of advanced analytic techniques, including artificial intelligence, rapid insights and analyses of cancer treatment and survival patterns will become more readily available. Such data will help clinicians better understand how their patients are treated, 
and what their outcomes are. Responsible stewardship of patient data, including appropriate safeguards to protect patient confidentiality, will be key in realizing this potential.

\section{Summary points}

- In the UK, there remain limited options in existing data for evaluating research questions related to oncology therapy.

- ChemoCare ${ }^{\circledR}$ is a prescription platform used for chemotherapy prescribing and patient management in over $80 \%$ of the UK's NHS cancer centers and has been in use in the NHS for over 15 years.

- This pilot study collected data from 37 patients at one hospital site (Singleton Hospital) and compared the congruence of data extracted directly from the prescription platform versus the gold standard comparison of a traditional medical chart review.

- The study demonstrated that this particular prescription platform is a valid data source for investigating research questions related to oncology treatment patterns and outcomes.

- There was a high concordance between the prescription platform and information taken directly from medical notes, including demographic variables, disease characteristics, systemic treatment patterns, treatment discontinuation and survival status.

- Direct hospital electronic medical record export is less prone to abstraction error, and it may be possible to obtain larger sample sizes using this method compared with a traditional chart review.

- This pilot study can be used as a framework for future real-world data studies using direct electronic medical record extraction.

\section{Author contributions}

D Stein, R Carroll and S Ramagopalan conceived the project and developed the study design with critical input from J Wagstaff. A Schultze conducted the analyses. A Schultze, M Soni and L McDonald wrote the paper. All authors reviewed the paper critically and approved its submission.

\section{Acknowledgments}

We would like to acknowledge the hard work of local research staff from Singleton Hospital and CIS Oncology who also contributed to this pilot study, as well as the contributions of Hankyul Kim, Evidera, who assisted with initial analytical work.

\section{Financial \& competing interests disclosure}

This study was sponsored by Bristol-Myers Squibb. L McDonald, R Carroll, S Ramagopalan are employees of Bristol-Myers Squibb; D Stein, M Soni and A Schultze are employees of Evidera that received funding from Bristol-Myers Squibb to conduct this study. J Wagstaff was the lead investigator from Singleton Hospital that received honoraria from Bristol-Myers Squibb for the conduct of the study. The authors have no other relevant affiliations or financial involvement with any organization or entity with a financial interest in or financial conflict with the subject matter or materials discussed in the manuscript apart from those disclosed.

No writing assistance was utilized in the production of this manuscript.

\section{Ethical conduct of research}

The authors state that the study was conducted in accordance with the ethical principles that have their origin in the Declaration of Helsinki, that are consistent with the current guidelines for International Society for Pharmacoepidemiology (ISPE) Good Pharmacoepidemiology Practices (GPP) and in accordance with local applicable laws and regulations. The protocol and proposed conduct of this chart review study (including the pilot study) were approved by an independent research ethics committee (REC): London - Dulwich Research Ethics Committee, by proportionate review. The study documentation was also reviewed by NHS-HRA, who approved this research involving patients in NHS organizations located in England. For patients in the chart review study from Wales, local governance review for local approval was performed by Health and Care Research Wales, and for Northern Ireland by HSC R\&D Application Gateway. For the chart review study, the required approvals from independent review committees, regulatory authorities and/or other local governance bodies were obtained before study initiation at any site. For the pilot study, data extraction took place after the site provided Caldicott Guardian approval for data release and necessary HRA and ethics approvals were obtained. 


\section{Open access}

This work is licensed under the Attribution-NonCommercial-NoDerivatives 4.0 Unported License. To view a copy of this license, visit http://creativecommons.org/licenses/by-nc-nd/4.0/

\section{References}

Papers of special note have been highlighted as: $\bullet$ of interest; $\bullet \bullet$ of considerable interest

1. CIS Oncology. About CIS. www.cis-healthcare.com/about-cis/

2. Berger ML, Curtis MD, Smith G, Harnett J, Abernethy AP. Opportunities and challenges in leveraging electronic health record data in oncology. Future Oncol. 12(10), 1261-1274 (2016).

-• This recent review summarizes key challenges of using electronic health records data, many of which are applicable also for our study.

3. Brundin-Mather R, Soo A, Zuege DJ et al. Secondary EMR data for quality improvement and research: a comparison of manual and electronic data collection from an integrated critical care electronic medical record system. J. Critical Care 47, 47295-47301 (2018).

- This recent study applies a similar methodology to ours, although in a different therapeutic area.

4. Knake LA, Ahuja M, McDonald EL et al. Quality of EHR data extractions for studies of preterm birth in a tertiary care center: guidelines for obtaining reliable data. BMC Pediatrics 16(1), 59 (2016).

5. Vandenberghe HE, Van Casteren V, Jonckheer P et al. Collecting information on the quality of prescribing in primary care using semi-automatic data extraction from GPs' electronic medical records. Int. J. Med. Inform. 74(5), 367-376 (2005). 
